\title{
UNCERTAINTY ESTIMATION OF AXES DIRECTION DETERMINATION OF INDUSTRIAL ROBOT USING AN ELLIPSOID CONCENTRATION MODEL
}

\author{
Andrei Vorotnikov, Elena Romash, Alexander Isaev, Olga Bashevskaya, \\ Giacomo Bianchi \& Yuri Poduraev
}
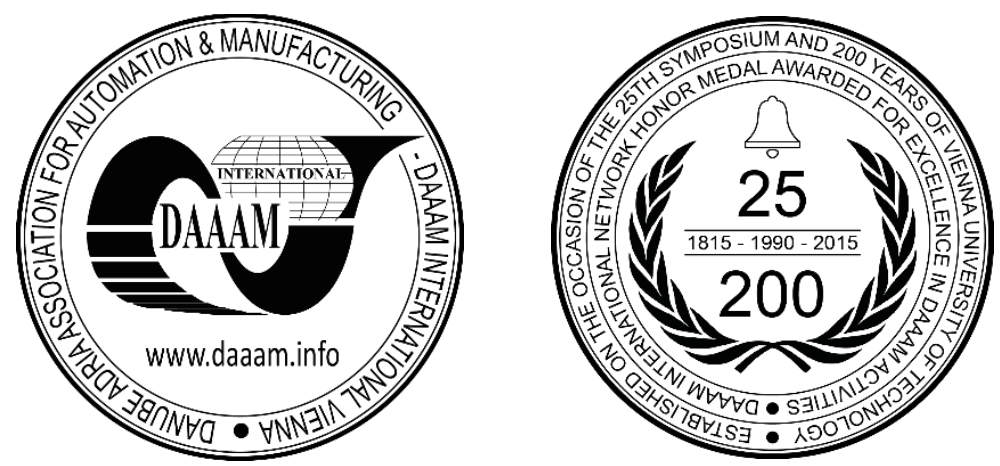

This Publication has to be referred as: Vorotnikov, A[ndrei]; Romash, E[lena]; Isaev, A[lexander]; Bashevskaya, O[lga]; Bianchi, G[iacomo] \& Poduraev, Y[uri] (2016). Uncertainty Estimation of Axes Direction Determination of Industrial Robot Using an Ellipsoid Concentration Model, Proceedings of the 27th DAAAM International Symposium, pp.0480-0486, B. Katalinic (Ed.), Published by DAAAM International, ISBN 978-3-902734-08-2, ISSN 1726-9679, Vienna, Austria

DOI: $10.2507 / 27$ th.daaam.proceedings.072

\begin{abstract}
There is uncertainty while measure different geometrical elements of industrial robots, multi-axis machines or other complex mechanisms during their calibration using CMM. This uncertainty has a large number of components. It is extremely difficult to estimate this uncertainty using ISO [12]. This article represents approach for uncertainty estimation of axes direction determination of industrial robot.

We revealed the concentration ellipsoid - a geometrical view of the spatial spread of error after repeated axis determination of an industrial robot. The mathematical model of the concentration ellipsoid was developed. In this paper we have analysed the big axis value of the concentration ellipsoid using a 3D surface. An iterative uncertainty search algorithm for the industrial robot's axis and its exit criterion was developed. Uncertainty of each axis of the industrial robot was estimated. The standard deviation of the iterative algorithm of uncertainty was calculated. The dependence of uncertainty from increasing the rotation radius of the measuring device reflector was experimentally revealed.
\end{abstract}

Keywords: concentration ellipsoid; uncertainty estimation; laser tracker; industrial robot calibration.

\section{Introduction}

Nowadays many studies in the field of mechatronics are related to the use of portable coordinate measuring machines (CMM). CMM are usually used for increasing accuracy of mechatronic devices. That promotes evolution of mechatronics [1] and confirms the importance of the direction of this article [13-15]. Laser tracker LTD 800 that was applied in this research is used in mechatronics for axis position determination of different mechanisms in the implementation of the following movements: linear, rotary or complex. For example the laser tracker provides calibration of industrial robots $[2,3]$, to determine the spindle axis position of the tool [4-6] or setup angular orientation of optical schemes for highspeed-shooting mechatronic systems of fast moving targets $[7,8]$.

In our research the KUKA KR16 - industrial robot with 6 joints was used as the measuring object. Each joint has its own axis of rotation. There is task when calibrate the industrial robot - to determine of all axes position in laser tracker reference frame. For determining the axis position of the industrial robot it is necessary to determine a point on the axis 
(joint center of rotation) and the direction (orientation) of this axis. Estimation of error in determining the joint center of rotation is represented in [2]. This article is devoted to uncertainty estimation of axes direction determination of an industrial robot.

CPA method was applied for measuring axis direction of an industrial robot. The description of CPA is represented in [2]. There is an uncertainty during measurement of the axis direction of an industrial robot. This uncertainty has a lot of components. During the research three main factors of uncertainty were revealed, the cumulative effect of which brings the greatest error in the result of uncertainty estimation:

- First, it is the error of the data processing algorithm of the coordinate points that were received on geometrical figures with small dimensions. The well-known example is fitting the data points that lie on a circle. When the data points form a small arc the determination of coordinates of the circle centre is not accurate [9]. In our research the similar problem is found in the approximation of coordinates of points on a plane: the ability to accurately determine the direction of the axis of the robot is lost on small arcs.

- Second, is the error of industrial robot caused by design features of the mechanism: flexibility; splits in kinematic pairs; temperature deformations in links of the robot $[10,11]$ and an input effect error of the servo drives [18, 19].

- Third, is the repeatability of the CMM that is rising during increasing of the distance between the laser tracker and the measuring object, and errors caused by features of the scanning mode.

So, there are three factors with many components that influence the estimation of uncertainty: error of the data processing algorithm; error anisotropy that is typical for industrial robots and also repeatability of the CMM. The calculation of uncertainty estimation represented in [12] has many components and is extremely difficult. Because of that, this article shows an iterative algorithm for searching uncertainty with the concentration ellipsoid model [16, 17]. Using of this algorithm helps to estimate the uncertainty when determining the axis of an industrial robot. The calculated uncertainty value can be used for analyzing and modeling. Also it should be noted that in this research the systematic error of a laser tracker was not taken into account for uncertainty. Only the dispersion in the conditions of repeatability was taken into account, since it is assumed that the systematic error was eliminated by other methods.

\section{Experiment about spatial spread of error}

First of all, we determine the geometrical view of spatial spread of errors during multiple determination of direction of the same axis of the industrial robot. It gives the concentration ellipsoid illustrated on figure 1. Based on the concentration ellipsoid an iterative algorithm for searching uncertainty was developed. This algorithm takes all factors with many components of uncertainties described above into account. For illustration of the concentration ellipsoid during multiple determination of the direction of the $5^{\text {th }}$ axis $(\overline{\mathbf{n}})$ of KUKA KR 16 next experiment was carried out: during changing of the joint at an angle $\pi \backslash 3$, the flange with mounted reflector of the laser tracker rotated around the $5^{\text {th }}$ axis while the laser tracker was scanning the trajectory $\left(\mathrm{x}_{i j}, y_{i j}, z_{i j}\right)$ of that rotation. It is evident that the trajectory of the reflector rotation is a circle that lies at plane $\alpha$. For collecting the statistical data, 51 rotations $(j=1 . . M, M=51)$ in one direction were made. Each rotation contains 500 scan points $(i=1 . . N, N=500)$.

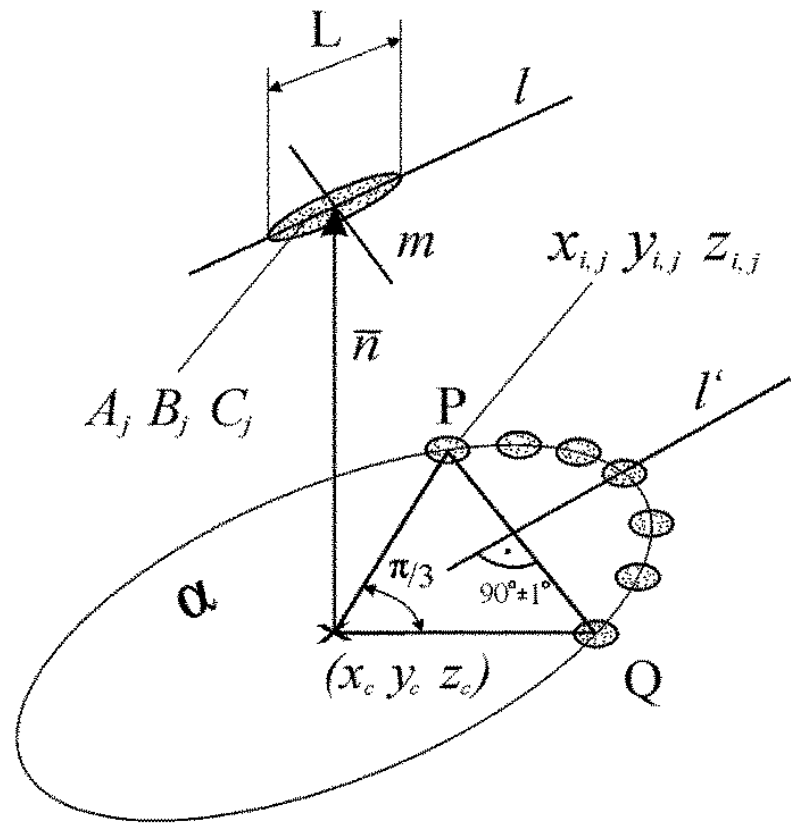

Fig. 1. Concentration ellipsoid determination. 
Each coordinates selection (j) of motion trajectory for determining of the $5^{\text {th }}$ axis parameters was fitted obtaining plane parameters $\left(A_{j}{ }^{\prime}, B_{j}{ }^{\prime}, D_{j}{ }^{\prime}\right)$ using the next formula:

$$
\left(\begin{array}{c}
A^{\prime}{ }_{j} \\
B^{\prime}{ }_{j} \\
D^{\prime}{ }_{j}
\end{array}\right)=\left(\begin{array}{ccc}
\sum_{i=1}^{N}\left(x_{i, j}+\delta x_{i j}\right)^{2} & \sum_{i=1}^{N}\left(x_{i, j}+\delta x_{i j}\right) \cdot y_{i, j} & \sum_{i=1}^{N}\left(x_{i, j}+\delta x_{i j}\right) \\
\sum_{i=1}^{N}\left(x_{i, j}+\delta x_{i j}\right) \cdot\left(y_{i, j}+\delta y_{i j}\right) & \sum_{i=1}^{N}\left(y_{i, j}+\delta y_{i j}\right)^{2} & \sum_{i=1}^{N}\left(y_{i, j}+\delta y_{i j}\right) \\
\sum_{i=1}^{N}\left(x_{i, j}+\delta x_{i j}\right) & \sum_{i=1}^{N}\left(y_{i, j}+\delta y_{i j}\right) & N
\end{array}\right) \cdot\left(\begin{array}{c}
\sum_{i=1}^{N}\left(x_{i, j}+\delta x_{i j}\right) \cdot\left(z_{i, j}+\delta z_{i j}\right) \\
\sum_{i=1}^{N}\left(y_{i, j}+\delta y_{i j}\right) \cdot\left(z_{i, j}+\delta z_{i j}\right) \\
\sum_{i=1}^{N}\left(z_{i, j}+\delta z_{i j}\right)
\end{array}\right)
$$

Where $\left(\delta \mathrm{x}_{i j}, \delta y_{i j}, \delta z_{i j}\right)$ - the uncertainty when measuring each coordinate (it is unknown in the experimental stage). Changing to parameters of axis direction $\left(a_{j}, b_{j}, c_{j}\right)$ with formula:

$$
\left(\begin{array}{l}
a_{j} \\
b_{j} \\
c_{j}
\end{array}\right)=\cdot\left(\begin{array}{c}
A^{\prime}{ }_{j} / \mathrm{D}_{j}^{\prime} \\
B_{j}^{\prime} / \mathrm{D}_{j}^{\prime} \\
1 / \mathrm{D}^{\prime}{ }_{j}
\end{array}\right)
$$

Translation them to unit vector using Euclidean norm:

$$
\left(\begin{array}{l}
A_{j} \\
B_{j} \\
C_{j}
\end{array}\right)=\cdot\left(\begin{array}{l}
a_{j} / \sqrt{a_{j}^{2}+b_{j}^{2}+c_{j}^{2}} \\
b_{j} / \sqrt{a_{j}^{2}+b_{j}^{2}+c_{j}^{2}} \\
c_{j} / \sqrt{a_{j}^{2}+b_{j}^{2}+c_{j}^{2}}
\end{array}\right)
$$

It is necessary to avoid error of the data processing algorithm of (1-3) at fitting when the trajectory points are very close to Cartesian axes (OX, OY, OZ) of the laser tracker coordinate system. We use special technique: two rotations of the laser tracker coordinate system according to the expression:

$$
\left(\begin{array}{l}
\mathrm{x}_{i, j}^{\prime} \\
y_{i, j}^{\prime} \\
z_{i, j}^{\prime}
\end{array}\right)=\left(\begin{array}{ccc}
\cos \left(\alpha_{k}\right) & 0 & -\sin \left(\alpha_{k}\right) \\
0 & 1 & 0 \\
\sin \left(\alpha_{k}\right) & 0 & \cos \left(\alpha_{k}\right)
\end{array}\right) \cdot\left(\begin{array}{ccc}
1 & 0 & 0 \\
0 & \cos \left(\alpha_{k}\right) & -\sin \left(\alpha_{k}\right) \\
0 & \sin \left(\alpha_{k}\right) & \cos \left(\alpha_{k}\right)
\end{array}\right) \cdot\left(\begin{array}{ccc}
\cos \left(\alpha_{k}\right) & -\sin \left(\alpha_{k}\right) & 0 \\
-\sin \left(\alpha_{k}\right) & \cos \left(\alpha_{k}\right) & 0 \\
0 & 0 & 1
\end{array}\right) \cdot\left(\begin{array}{l}
\mathrm{x}_{i, j} \\
y_{i, j} \\
z_{i, j}
\end{array}\right)
$$

Where the angle of rotation is $\alpha_{k},(k=1 . .2)$.The first rotation is 30 degrees, the second is 45 degrees. After each rotation the unit vector of the fitted plane $(j=1)$ is determined. Given unit vectors after fitting are turned back. Than three vectors: two after rotation and fitting, and one just fitting. Than three vectors are comparing among themselves. If a significant discrepancy between the three fitted vectors is detected, they are being compared, one of the three is excluded from the maximum mathematical error, and the coordinates are selected from any rotation of the remaining two. It should be noted that this problem shows only when the fitted axis is very close to Cartesian axes (OX, OY, OZ). The received set of unit vectors has a geometrical form of an ellipsoid with two axes ( $l$ (big axis) and $m$ (small axis)), as it is shown on figure 1 . The third axis of the ellipsoid is located along $(\overline{\mathbf{n}})$ and is negligible.

\section{Mathematical model of the concentration ellipsoid}

After receiving the geometrical view of the spatial spread of errors during multiple determination of direction of the same axis of the industrial robot we made the decision to develop a mathematical model of the concentration ellipsoid. The mathematical model of the concentration ellipsoid is needed for determining the dimension of $L$ on axis $l$ that depends on the number of points $(\mathrm{N})$ on the arc and also depends on radius of reflector rotation $(\mathrm{R})$. There is an assumption in the basis of model: each $\mathrm{i}$-point on the arc $\left(\left(\mathrm{x}_{i},\right)_{j},\left(y_{i},\right)_{j},\left(z_{i},\right)_{j}\right)$ has normal distribution with equal mean square deviations in Cartesian coordinates $\left(\sigma / \sqrt{3}=\sigma_{x}=\sigma_{y}=\sigma_{z}\right)$ because the geometrical view of spatial spread of errors in each i-point is unknown:

$$
\sigma=\sqrt{\sigma_{x}^{2}+\sigma_{y}^{2}+\sigma_{z}^{2}}
$$


The rotation of reflector is imitated by next formula:

$$
\left(\begin{array}{c}
\mathrm{x}_{m i, j} \\
y_{m i, j} \\
z_{m i, j}
\end{array}\right)=\left(\begin{array}{c}
\mathrm{x}_{c}+R \cdot \cos \left(\frac{\pi \cdot i}{v \cdot N}\right)+\sigma_{x i} \\
y_{c}+R \cdot \cos \left(\frac{\pi \cdot i}{v \cdot N}\right)+\sigma_{y i} \\
z_{c}+\sigma_{z i}
\end{array}\right)_{j}
$$

Where $\left(x_{m i, j}, y_{m i, j}, z_{m i, j}\right)$ - are the coordinates of model of reflector motion trajectory $(i=1 . . N$ (number of points on $\operatorname{arc}$ ), $j=1 . . M$ (number of arcs)), $\left(x_{c}, y_{c}, z_{c}\right)$ - coordinates of center rotation, $R$ - radius of reflector rotation, $v$ - number, corresponding to the value of arc. It is necessary to rotate the coordinate frame according to (4) for obtaining coordinates of model of reflector motion trajectory. For determining the unit vectors $\left(A_{m j}, B_{m j}, C_{m j}\right)$ of the concentration ellipsoid model it is necessary to use (1-3).

Using the ellipsoid concentration model it is possible to receive ellipsoids with different $L$ that depend on changing parameters of the model. It is reasonable to characterize several dependences: if the arc is more than $\pi$, the geometrical form of the ellipsoid changes to a disk, if the arc less than $\pi$, the ellipsoid is formed. Very small arc length significantly increases the dimensions of $L$ of the ellipsoid. If the mean square deviation (uncertainty) changes, is almost proportionally changes the ellipsoid dimensions. The main advantage of the received concentration ellipsoid (model) is a knowledge about the projection of the ellipsoid big axis $l^{\prime}$ on plane $\alpha$. It is always perpendicular (with deviation about \pm 1 degree) to the vector PQ, as illustrated on figure 1. This is a key feature for robotics and mechatronics. Movement of the measuring area (the measured arc) allows to change the direction of the spatial spread of error $l$.

Applying the mathematical model represented by (1) - (5) we constructed the 3D surface illustrated on figure 2, which shows the $L$ dimension dependence of the concentration ellipsoid from number of points $(\mathrm{N})$ on arc and from radius of reflector rotation $(\mathrm{R})$. The graph of a 3D surface shows the size L considerably increasing at small values of the radius of reflector rotation and small amount of points on an arc. This 3D surface also shows behavior of the mathematical model of concentration ellipsoid with mean square deviation of $0.0375 \mathrm{~mm}$ on a $\pi / 3$ arc.

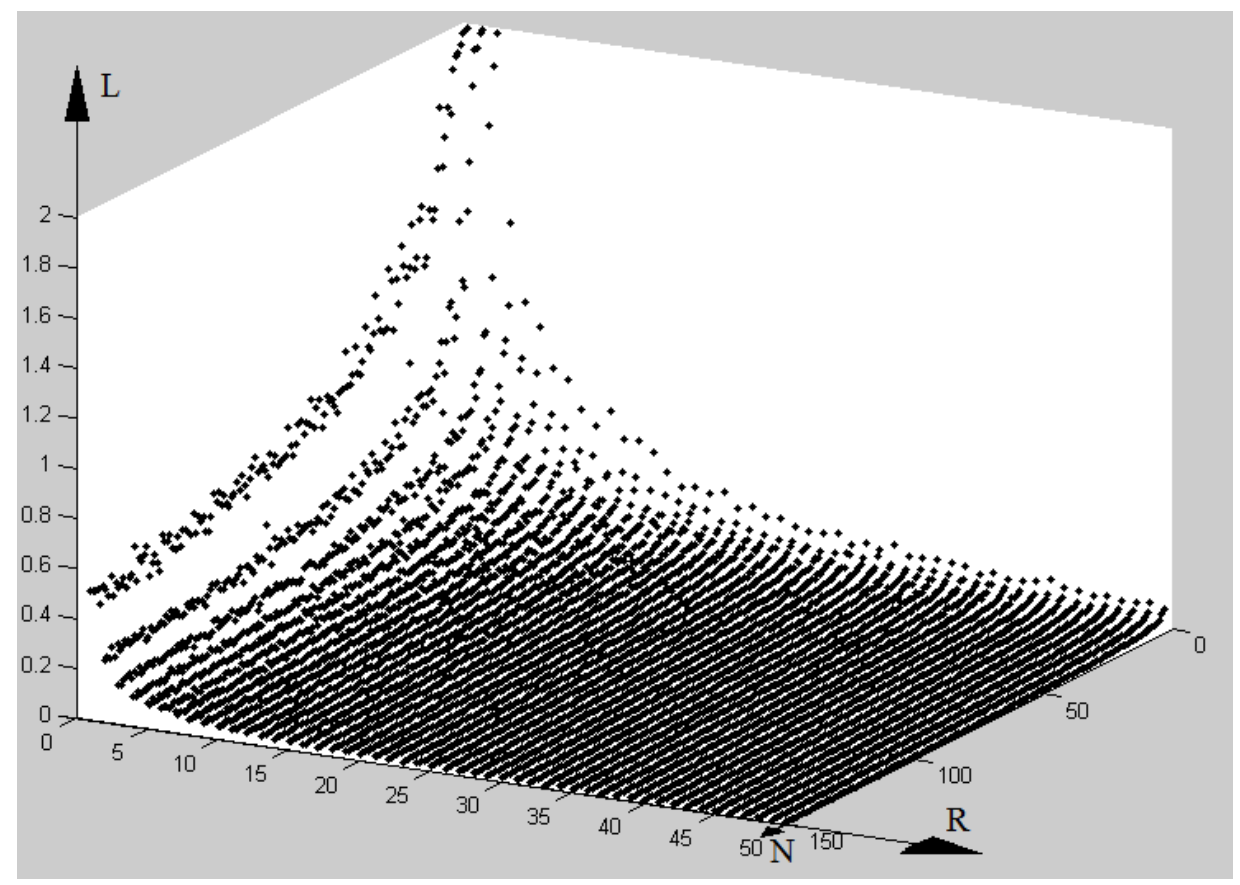

Fig. 2. 3D surface that shows the behaviour of big axis $(L)$ of concentration ellipsoid.

\section{Iterative uncertainty search algorithm}

Iterative algorithm for searching uncertainty is shown on figure 3. The algorithm is based on the mathematical model of the concentration ellipsoid (1) - (5) described above. The initial value of uncertainty is chosen as $0.001 \mathrm{~mm}$. The increase in value of uncertainty occurs with some step the amount of which is chosen empirically, as a reference serves the step number and the final operating time of the algorithm. At small steps the operating time of an algorithm increases considerably, at big steps the accuracy of searching the uncertainty decreases. 


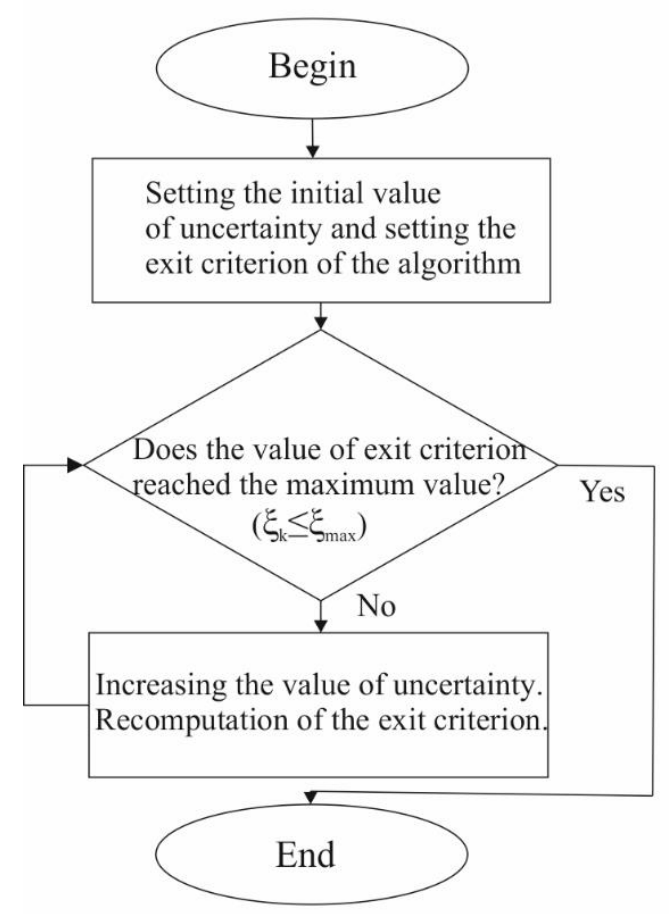

Fig. 3. Iterative algorithm for searching uncertainty.

The exit criterion of iterative algorithm is the mean square deviation $\xi_{k}$, where $k$ is the number of algorithm iteration:

$$
\xi_{k}=\sqrt{\frac{1}{M^{2}} \cdot \sum_{p=1}^{M^{2}}\left(\mathrm{~d}_{p}-\mathrm{d}_{m i d}\right)^{2}}
$$

Where $d_{p}$ - are distances between the received points $\left(A_{j}, B_{j}, C_{j}\right)$ (or $\left.\left(A_{m j}, B_{m j}, C_{m j}\right)\right)$, that create an ellipsoid and characterize the uncertainty $(p=1 . . M \cdot M) . d_{\text {mid }}$ - mean value of samples of $d_{p} \cdot \xi_{\max }$ determines after measuring and receiving the concentration ellipsoid. Recalculation of the exit criterion (mean square deviation $\xi_{k}$ ) occurs according to the formulas (1) - (6) by means of creation of the concentration ellipsoid.

The result of the algorithm work is presented in table 1. The row mathematical model of concentration ellipsoid (1) (5) shows ellipsoid model parameters. The values which are the result of work of the algorithm are represented in row value of $\sigma_{A}$. Also, in the corresponding row, standard deviations from $\sigma_{A}$ are estimated.

\begin{tabular}{|c|c|c|c|c|c|}
\hline $\begin{array}{c}\text { Mathematical } \\
\text { model of } \\
\text { concentration } \\
\text { ellipsoid }\end{array}$ & $\begin{array}{c}\mathrm{R}=20, \mathrm{~mm} \\
\mathrm{~N}=150 \\
\mathrm{M}=100 \\
\sigma=0.01, \mathrm{~mm}\end{array}$ & $\begin{array}{c}\mathrm{R}=50, \mathrm{~mm} \\
\mathrm{~N}=500 \\
\mathrm{M}=15\end{array}$ & $\begin{array}{c}\mathrm{R}=160, \mathrm{~mm} \\
\mathrm{\sigma}=300 \\
\mathrm{M}=25\end{array}$ & $\begin{array}{c}\mathrm{R}=300, \mathrm{~mm} \\
\mathrm{~N}=500 \\
\mathrm{M}=200\end{array}$ & $\begin{array}{c}\mathrm{R}=700, \mathrm{~mm} \\
\mathrm{~N}=300 \\
\mathrm{M}=50\end{array}$ \\
\hline Value of $\sigma_{A}$ & 0.011 & $0.075, \mathrm{~mm}$ & $0.25, \mathrm{~mm}$ & $\sigma=0.05, \mathrm{~mm}$ \\
\hline $\begin{array}{c}\text { Standard deviations } \\
\text { from } \sigma_{A}\end{array}$ & \pm 0.0005 & \pm 0.01 & \pm 0.0025 & 0.245 & 0.0495 \\
\hline
\end{tabular}

Table 1. Results of work of iterative algorithm for searching uncertainty using data of concentration ellipsoid model.

Fluctuations and systematic errors of values $\sigma_{A}$ from model uncertainty $\sigma$ are caused by the choice of exit criterion of the algorithm. Different exit criterion give different results from the algorithm. The best results are obtained by applying the algorithm at a value $M=25 ; 50$. For estimation of real values of uncertainty the experiment with the industrial robot KUKA KR 16 was made. For all joints individually all motion trajectories of the reflector fixed on a robot flange were scanned. A set of coordinates for each circle consisted of 300 points. For receiving the concentration ellipsoid of uncertainty each circle was received 25 times. Results of the experiment are represented in table 2 . 


\begin{tabular}{|c|c|c|c|c|c|c|}
\hline $\begin{array}{c}\text { Number of joint } \\
\text { (axis of industrial } \\
\text { robot) }\end{array}$ & 1 & 2 & 3 & 4 & 5 & 6 \\
\hline $\begin{array}{c}\text { Radius of reflector } \\
\text { rotation R }\end{array}$ & 1115.780 & 1071.275 & 854.973 & 24.704 & 184.542 & 24.797 \\
\hline Uncertainty $\sigma$ & 0.030 & 0.016 & 0.007 & 0.053 & 0.005 & 0.051 \\
\hline
\end{tabular}

Table 2. Results of work of the iterative algorithm for searching uncertainty using experimental data.

It is possible to do the following conclusions using experimental data: uncertainties have big values on small radiuses, therefore it is reasonable to increase rotation radius for finding of the $4^{\text {th }}$ and $6^{\text {th }}$ axes of industrial robots by additional equipment. The development of a special calibration device is a next step of our research. The tendency of increasing uncertainty on big circles was found. That could be also taken into account for further mathematical modelling of the concentration ellipsoid. Because of insufficient quantity of experimental data, the dependence of uncertainty from the radius of reflector rotation was fitted with a considerable error, but has the functional dependence close to:

$$
\sigma_{A}=\frac{f}{R}+g \cdot R^{2}+h \cdot R+e
$$

Where $f, g, h, e$ - are the parameters of dependence of uncertainty from the radius of the reflector rotation.

\section{Conclusion}

There is uncertainty when measuring different geometrical elements of industrial robots, multi-axis machines or other complex mechanisms for their calibration using CMM. This uncertainty has a large number of components. It is extremely difficult to estimate this uncertainty using ISO [12]. This article represents approach for uncertainty estimation of axes direction determination of industrial robot.

We revealed the concentration ellipsoid - a geometrical view of the spatial spread of error after repeated axis determination of an industrial robot. The mathematical model of the concentration ellipsoid was developed. In this paper using a 3D surface we have analysed the dimension of the big axis of the concentration ellipsoid that depends on number of points on arc and also on radius of reflector rotation. An iterative uncertainty search algorithm for the industrial robot axis and its exit criterion was developed. Uncertainty of each axis of the industrial robot was estimated. The standard deviation of the iterative algorithm was calculated. The dependence of uncertainty from increasing the rotation radius of the measuring device reflector was experimentally revealed. This dependence has influence on optimal measurement area. By results of the experiments and by results of the work of the algorithm it is recommended to carry out the uncertainty estimation when calibrating the industrial robot or a mechatronic device since values of uncertainty for various axes will be different. It is also reasonable to develop and use the specialized equipment for decreasing the uncertainty in case of determination of axes of industrial robots. The development of specialized equipment is the purpose of further research. Also, it is reasonable to carry out similar estimation of uncertainty for manufacturers of the CMM to provide better performance of their equipment.

\section{Acknowledgments}

The reported study was partially supported by RFBR, research project №15-58-78024 Итал_а

\section{References}

[1] Poduraev, Yu. V. (2006). Mechatronics: fundamentals, methods, instrumentation, M.: Engineering, ISBN 5-21703355-X, Moscow.

[2] Vorotnikov, A. A., Poduraev, Yu. V., Romash E.V. (2015) Estimation of error in determining the centers of rotation of links in a kinematic chain for industrial robot calibration techniques, Measurement Techniques, Vol. 58, No. 8, November, 2015, pp. 864-871. DOI: 10.1007/s11018-015-0809-9

[3] Vorotnikov, A[ndrei]; Bashevskaya, O[lga]; Ilyukhin, Y[uri]; Romash, E[lena]; Isaev, A[.] V[.] \& Poduraev, Y[uri] (2016). Geometrical Approach for Industrial Robot Axis Calibration Using Laser Tracker, Proceedings of the 26th DAAAM International Symposium, pp.0897-0904, B. Katalinic (Ed.), Published by DAAAM International, ISBN 978-3-902734-07-5, ISSN 1726-9679, Vienna, Austria. DOI: 10.2507/26th.daaam.proceedings.125

[4] Grechishnikov, V. A., Petukhov, Yu. E., Pivkin, P. M., A. V. Isaev, V. B. Romanov, and P. V. Domnin. Lathe Turning of Complex Shaped Parts Providing Desired Surface Microrelief / Russian Engineering Research, 2016, Vol. 36, No. 3, pp. 229-231. ISSN 1068-798X DOI: 10.3103/S1068798X16030059 
[5] Grechishnikov, V. A., Isaev, A. V., Ilyukhin, Yu. V., Pivkin, P. M., Vorotnikov, A. A., Kharchenko, A. N., Bianchi, G., Leonesio, M., Pedrocchi, N., Molinari, Robotic cells for machinning and system of their instrumental providing. M.: FGBOU IN MSTU OF STANKIN, 2015, ISBN 978-5-7028-0683-9, Moscow.

[6] Grechishnikov, V. A., Isaev, A. V., Ilyukhin, Yu. V., Pivkin, P. M., Vorotnikov, A. A., Kharchenko, A. N., Bianchi, G., Leonesio, M., Pedrocchi, N., Molinari, T. L., Building principles of robotic machining systems and their cutting tool system, Vestnik MSTU «STANKIN», №4 (35), 2015. pp. 46-51, ISSN 2072-3172.

[7] D. Klimov, Y. Poduraev, Design and Experimental Testing of a Robotic System for High Speed Recording, in: Annals of DAAAM for 2014 \& Proceedings of the 25th International DAAAM Symposium, ISSN 1877-7058, ISBN 978-3-901509-97-1, Editor B[ranko] Katalinic, Published by DAAAM International, Vienna, Austria, 2014, pp. $1332-1337$.

[8] Klimov, D[aniil] \& Poduraev, Y[ury] (2016). Robotic Flight Follower Systemfor High Speed Recording, Proceedings of the 26th DAAAM International Symposium, pp.0720-0726, B. Katalinic (Ed.),Published by DAAAM International, ISBN 978-3-902734-07-5, ISSN 1726-9679, Vienna, Austria

[9] Chernov, N., (2011). Circular and linear regression fitting circles and lines by least squares. CRC Press Taylor \& Francis Group, ISBN 978-1-4398-3590-6, USA.

[10] Bashevskaya O.S., Gulieva R.M., Romash E.V., Melnichenco E.A. Research of temperature deformations of end bar. Vestnik MSTU «STANKIN», №4. pp. 36-40.ISSN 2072-3172.

[11] Bashevskaya, O. S. Bushuev, S. V., Poduraev, Yu. V., Koval'skii, M. G., Kainer, G. B., Romash E. V. An Investigation of the Influence of Temperature Deformations on the Precision of Linear Measurements, Measurement Techniques, Vol. 56, December, 2013, Issue 9, pp 1016-1020, DOI: 10.1007/s11018-013-0322-y

[12] ISO/IEC Guide 98-3:2008 - Uncertainty of measurement

[13] Nubiola, A., Bonev, I. A.. Absolute calibration of an ABB IRB 1600 robot using a laser tracker. Robotics and Computer-Integrated Manufacturing, Vol. 29 (1), February, 2013, pp. 236-245, DOI: 10.1016/j.rcim.2012.06.004

[14] Yahui Gan, Xianzhong Dai, and Donghui Dong. Robot Calibration for Cooperative Process under Typical Installation, Available from: http://dx.doi.org/10.1155/2014/576420

[15] Klimchik A., Pashkevich A., Chablat D.,Hovland G. Compliance error compensation technique for parallel robots composed of non-perfect serial chains, Available from: http://dx.doi.org/10.1016/j.rcim.2012.09.008

[16] Kramer, H. (1975). Mathematical methods in statistics, M.:Inostranaya literature, Moscow.

[17] Belyaev, Yu.K., Chepurin, E.E., (1982) The basis of statistics, M.: Moscow University Publisher, Moscow.

[18] Ilyukhin, Y[uri] \& Kolesnichenko, R[uslan] (2016). Impedance Control of High-Precision Geared Servo Drives with Two Motors for Technological Robots, Proceedings of the 26th DAAAM International Symposium, pp.0599-0607, B. Katalinic (Ed.), Published by DAAAM International, ISBN 978-3-902734-07-5, ISSN 1726-9679, Vienna, Austria, DOI: 10.2507/26th.daaam.proceedings.081

[19] Ilyukhin Yu. V. Poduraev Yu. V. Improving the Precision of Mechatronic Robot Drives. // ISSN 1068_798X, Russian Engineering Research, 2016, Vol. 36, No. 4, pp. 328-334. (C) Allerton Press, Inc., 2016. Original Russian Text (C) Yu.V. Ilyukhin, Yu.V. Poduraev, 2015, published in STIN, 2015, No. 9, pp. 30-37. 